\title{
Broadband MC DS-CDMA Using Space-Time and Frequency-Domain Spreading
}

\author{
Lie-Liang Yang and Lajos Hanzo \\ Dept. of ECS, University of Southampton, SO17 1BJ, UK. \\ Tel: +44-23-8059 3125, Fax: +44-23-8059 4508 \\ Email: 1ly,lh@ecs.soton.ac.uk; http://www-mobile.ecs.soton.ac.uk
}

\begin{abstract}
In this contribution multicarrier direct-sequence codedivision multiple-access (MC DS-CDMA) using space-time spreading (STS) assisted transmit diversity and frequency-domain (F-domain) spreading is investigated in the context of broadband communications over frequency-selective Rayleigh fading channels. We consider the attainable capacity extension of broadband MC DS-CDMA with the advent of using Time-Frequency-domain (TF-domain) spreading. The BER performance of STS assisted broadband MC DS-CDMA using Binary Phase Shit Keying (BPSK) modulation and TF-domain spreading is investigated by simulation for a range of parameter values. Both the correlation based singleuser detector and the decorrelating multiuser detector are considered. Our study shows that the number of users supported by the broadband MC DS-CDMA system is determined by the product of the T-domain spreading factor and the F-domain spreading factor, while it is independent of the frequency diversity order. Furthermore, when multiuser detection assisted F-domain spreading is considered, the broadband MC DS-CDMA system is capable of supporting a substantially increased number of users, while maintaining a similar bit error ratio (BER) performance to that of the broadband MC DS-CDMA system using no F-domain spreading.
\end{abstract}

\section{INTRODUCTION}

Recently a considerable number of studies has been conducted in the context of the future wireless communication systems. Broadband multicarrier direct-sequence code-division multiple-access (MC DS-CDMA) constitutes one of the most promising multiple-access schemes and hence enjoyed wide attention. A broadband MC DSCDMA system using space-time spreading [1] assisted transmit diversity has been proposed and studied in [2]. It was shown that the capacity of broadband MC DS-CDMA systems can be substantially increased, when using multiple transmit antennas for providing spatial diversity. The achievable performance can be further enhanced by using frequency domain (F-domain) interleaving of the transmitted signals accross several subcarriers for the sake of providing frequency diversity.

However, for a given number of subcarriers, each having a constant chip rate, the number of bits per symbol and the processing gain determined by the number of chips per symbol decreases upon increasing the F-domain interleaving depth. Hence, the number of users supported by MC DS-CDMA also decreases upon increasing the Fdomain interleaving depth, i.e. upon increasing the frequency diversity order. This is because the number of DS spreading codes having good correlation characteristics is determined by the number of chips per symbol. Instead of the above-mentioned pure F-domain interleaving, in MC DS-CDMA the transmitted data stream can be spread in both the T-domain and the F-domain, i.e. in the TF-domain, in order to mitigate the problem that the number of users supported by MC DS-CDMA decreases upon increasing the F-domain interleaving depth. However, this is a topic rarely investigated so far in the literature. In this contribution we augment our work presented in [2] and

\footnotetext{
The support of the European Union and that of the EPSRC, UK is gratefully acknowledged.

VTC'2002, Vancouver, Canada
}

propose a broadband MC DS-CDMA scheme, which employs STS assisted transmit diversity and time-frequency-domain (TF-domain) spreading. Our study shows that the number of users supported by the broadband MC DS-CDMA system is determined by the product of the T-domain spreading factor and the F-domain spreading factor, while it is independent of the frequency diversity order. Furthermore, when multiuser detection assisted F-domain spreading is considered, the broadband MC DS-CDMA system is capable of supporting a substantially increased number of users, while maintaining a similar bit error ratio (BER) performance to that of broadband MC DS-CDMA system using no F-domain spreading.

\section{SYSTEM DESCRIPTION}

\section{A. Transmitter Model}

The system considered in this paper is an orthogonal MC DSCDMA scheme [3] using $U \cdot S$ number of subcarriers, $T_{x}$ number of transmitter antennas and one receiver antenna. Furthermore, in this paper a synchronous MC DS-CDMA scheme is investigated, where the $K$ user signals are transmitted synchronously. The transmitter schematic of the $k$ th user is shown in Fig.1, where real-valued data symbols using BPSK modulation and real-valued spreading [1] were considered. As shown in Fig.1, at the transmitter side a block of $U \cdot L_{x}$ data bits each having a bit duration of $T_{b}$ is S-P converted to $U$ parallel sub-blocks. Each parallel sub-block has $L_{x}$ data bits, which are space-time spread using the schemes of [1] with the aid of $M_{x}$ number of orthogonal spreading codes - for example Walsh codes - $\left\{c_{k, 1}^{I}(t), c_{k, 2}^{I}(t), \ldots, c_{k, M_{x}}^{I}(t)\right\}, k=1,2, \ldots, K$ and mapped to $T_{x}$ transmitter antennas. The symbol duration of the STS signals is $U L_{x} T_{b}$, and the discrete period of the orthogonal codes is $U L_{x} T_{b} / T_{c}=U L_{x} N$, where $N=T_{b} / T_{c}$ and $T_{c}$ represents the chip-duration of the orthogonal spreading codes. The orthogonal codes take the form of $c_{k, i}^{I}(t)=\sum_{j=0}^{U L_{x} N-1} c_{k, i}^{I}[j] \cdot \Psi_{T_{c}}\left(t-j T_{c}\right)$, where $c_{k, i}^{I}[j] \in\{+1,-1\}$ and they obey the orthogonality relationship of $\sum_{l=0}^{U L_{x} N} c_{i, m}[l] c_{j, n}[l]=0$, whenever $i \neq j$ or $m \neq n$. Furthermore, $\Psi_{T_{c}}(t)$ represents the chip impulse waveform defined over the interval of $\left[0, T_{c}\right)$. As seen in Fig.1, following STS, each STS block generates $T_{x}$ parallel signals to be mapped to the $T_{x}$ transmitter antennas. The specific $U$ STS signals of Fig.1, which are output by the $U$ STS blocks and which will be transmitted using the same antenna from the set $1,2, \ldots, T_{x}$ are then spread in the F-domain using an orthogonal sequence $\left\{c_{k}^{I I}[0], c_{k}^{I I}[1], \ldots, c_{k}^{I I}[S-1]\right\}$, so that each STS signal is transmitted on $S$ number of subcarriers, rather than one. The F-domain spreading guarantees that the same STS signal is transmitted by the specific $S$ subcarriers having the maximum possible frequency spacing, so that they experience independent fading and hence achieve maximum frequency domain diversity. Finally, as shown in Fig.1, the Inverse Fast Fourier Transform (IFFT) is invoked for carrying out multicarrier modulation, and the IFFT block's output signal is transmitted using one of the transmitter antennas. 


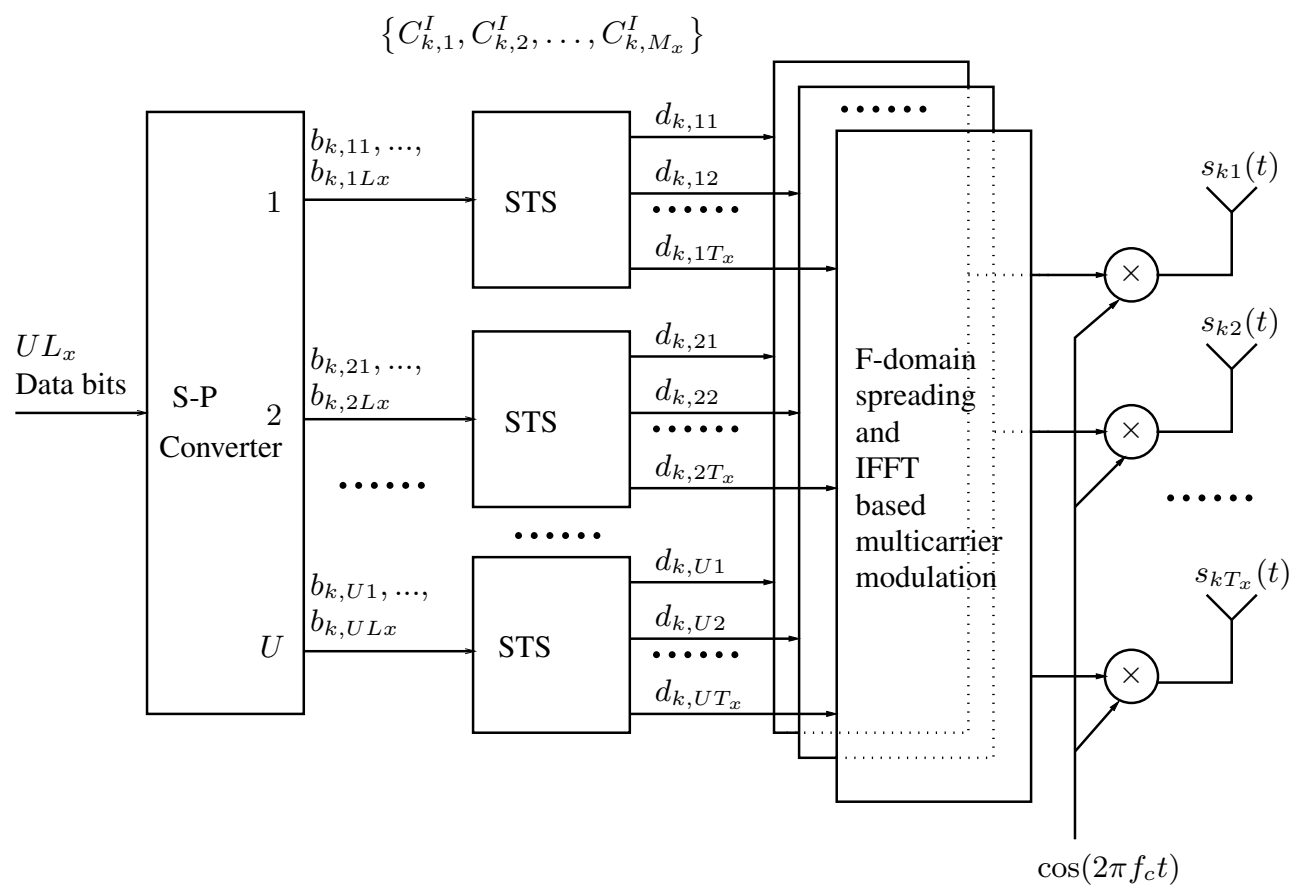

Fig. 1. The transmitter schematic of the MC DS-CDMA system using space-time spreading.

It can be shown that the signals transmitted from the $T_{x}$ number of transmitter antennas can be expressed as

$$
\begin{array}{r}
\mathbf{s}_{k}(t)=\operatorname{Re}\left\{\sqrt{\frac{2 E_{b}}{U T_{b}} \frac{1}{S M_{x} T_{x}}}\left[\mathbf{C}_{k} \mathbf{B}_{k}\right]^{T} \mathbf{Q w}\right. \\
\left.\times \exp \left(j 2 \pi f_{c} t\right)\right\},
\end{array}
$$

where $E_{b} / U T_{b}$ represents the transmitted power per subcarrier expressed as $L_{x} E_{b} / U L_{x} T_{b}=E_{b} / U T_{b}$. The factor $S$ in the denominator is due to the $S$-fold F-domain spreading, while the factor of $M_{x} T_{x}$ represents STS using $M_{x}$ number of orthogonal codes and $T_{x}$ number of transmitter antennas. In (1) $\mathbf{s}_{k}(t)=\left[\begin{array}{llll}s_{k 1}(t) & s_{k 2}(t) \ldots & s_{k T_{x}}(t)\end{array}\right]^{T}-$ where the superscript $T$ denotes the vector or matrix transpose - represents the transmitted signal vector of the $T_{x}$ transmitter antennas. The matrix $\mathbf{Q}$ in (1) represents the F-domain spreading, while is a $U \times U S$ dimensional matrix expressed as

$$
\mathbf{Q}=\left[\mathbf{C}_{k}^{I I}[0] \mathbf{C}_{k}^{I I}[1] \ldots \mathbf{C}_{k}^{I I}[S-1]\right]
$$

where $\mathbf{C}_{k}^{I I}[s], s=0,1, \ldots, S-1$ are diagonal matrices of rank $U$, which can be expressed as

$$
\mathbf{C}_{k}^{I I}[s]=\operatorname{diag}\left\{c_{k}^{I I}[s], c_{k}^{I I}[s], \ldots, c_{k}^{I I}[s]\right\}
$$

Furthermore, $\mathbf{C}_{k}$ is a $U \times U M_{x}$ dimensional matrix constituted by the orthogonal codes, which can be expressed as

$$
\mathbf{C}_{k}^{T}=\left(\begin{array}{cccc}
c_{k, 1}^{I}(t) & 0 & \ldots & 0 \\
c_{k, 2}^{I}(t) & 0 & \ldots & 0 \\
\vdots & \vdots & \ddots & \vdots \\
c_{k, M_{x}}^{I}(t) & 0 & \ldots & 0 \\
0 & c_{k, 1}^{I}(t) & \ldots & 0 \\
0 & c_{k, 2}^{I}(t) & \ldots & 0 \\
\vdots & \vdots & \ddots & \vdots \\
0 & c_{k, M_{x}}^{I}(t) & \ldots & 0 \\
\vdots & \vdots & \ddots & \vdots \\
0 & 0 & \vdots & c_{k, 1}^{I}(t) \\
0 & 0 & \vdots & c_{k, 2}^{I}(t) \\
\vdots & \vdots & \ddots & \vdots \\
0 & 0 & \vdots & c_{k, M_{x}}^{I}(t)
\end{array}\right) .
$$

To elabrate further, in (1) $\mathbf{B}_{k}$ is a $U M_{x} \times T_{x}$ dimensional matrix mapping the $U$ sub-block data bits to $T_{x}$ anttenas, according to the requirements of STS [1] as seen in Fig.1. Specifically, the matrix $\mathbf{B}_{k}$ can be expressed as

$$
\mathbf{B}_{k}=\left[\begin{array}{llll}
\mathbf{B}_{k 1}^{T} \mathbf{B}_{k 2}^{T} & \ldots & \mathbf{B}_{k U}^{T}
\end{array}\right]^{T}
$$

where $\mathbf{B}_{k u}$ for $u=1,2, \ldots, U$ are $M_{x} \times T_{x}$ dimensional matrices, which obey the structure of

$\mathbf{B}_{k u}=\left(\begin{array}{cccc}a_{11} b_{k, 11}^{\prime} & a_{12} b_{k, 12}^{\prime} & \ldots & a_{1 L_{x}} b_{k, 1 T_{x}}^{\prime} \\ a_{21} b_{k, 21}^{\prime} & a_{22} b_{k, 22}^{\prime} & \ldots & a_{2 L_{x}} b_{k, 2 T_{x}}^{\prime} \\ \vdots & \vdots & \ddots & \vdots \\ a_{M_{x} 1} b_{k, M_{x} 1}^{\prime} & a_{U 2} b_{k, M_{x} 2}^{\prime} & \ldots & a_{M_{x} L_{x}} b_{k, M_{x} T_{x}}^{\prime}\end{array}\right)$, 
where $a_{i j}$ represents the sign of the element at the $i$ th row and the $j$ th column, which is determined by the STS design rule, while $b_{k, i j}^{\prime}$ in $\mathbf{B}_{k u}$ is the data bit assigned to the $(i, j)$ th element, which is one of the $L_{x}$ input data bits $\left\{b_{k, u 1}, b_{k, u 2}, \ldots, b_{k L_{x}}\right\}$ of user $k$.

Finally, in (1) $\mathbf{w}$ represents the multicarrier modulated vector of length $U S$, which can be expressed as

$$
\mathbf{w}=\left[\begin{array}{llll}
\exp \left(j 2 \pi f_{1} t\right) & \exp \left(j 2 \pi f_{2} t\right) \ldots \exp \left(j 2 \pi f_{S U} t\right)
\end{array}\right]^{T} .
$$

Equation (1) represents the general form of the transmitted MC DSCDMA signals using STS, regardless of the values of $L_{x}, M_{x}$ and $T_{x}$. However, the study conducted in [1] has shown that only the class of STS schemes using the parameters of $L_{x}=M_{x}=T_{x}$ constitute attractive schemes, since they are capable of providing maximal transmit diversity without requiring extra STS codes. Hence, in this contribution we only investigate these attractive STS schemes.

The total number of users supported by the broadband MC DSCDMA system using TF-domain spreading and the assignment of orthogonal codes to the users is analyzed as follows. The total number of orthogonal codes that can be used for STS is $U L_{x} N$ and the maximum number of users supported by these orthogonal codes is $\mathcal{K}_{\max }=U L_{x} N / M_{x}$. By contrast, the total number of orthogonal codes that can be used for F-domain spreading is $S$. This implies that even if a set of $S$ number of users share the same set of STS codes, these $S$ user signals might be distinguishable with the aid of the associated $S$ number of F-domain spreading codes. Explicitly, the total number of users supported is $S \mathcal{K}_{\max }=U S L_{x} N / M_{x}$. Therefore, the orthogonal spreading codes can be assigned as follows. If the number of users is in the range of $0 \leq K \leq \mathcal{K}_{\max }$, these users will be assigned the required orthogonal STS codes and the same F-domain orthogonal spreading code. The resultant scheme is the same as the one we studied in [2]. However, when the number of users is in the range of $s \mathcal{K}_{\max } \leq K \leq(s+1) \mathcal{K}_{\max }, s=1,2 \ldots, S-1$, then the same set of $M_{x}$ number of STS orthogonal codes must be assigned to $s$ or $(s+1)$ users, but these $s$ or $(s+1)$ users are assigned different $\mathrm{F}$ domain spreading codes. These $s$ or $(s+1)$ users employing the same set of $M_{x}$ STS codes are identified by their corresponding F-domain spreading codes. Since the subcarrier signals across which F-domain spreading takes place encounter independent fading, the orthogonality of the F-domain spreading codes cannot be retained. Hence, multiuser interference is inevitably introduced, which degrades the BER performance, when increasing the number of users sharing the same set of $M_{x}$ number of STS orthogonal codes.

\section{B. Channel Model and System Parameter Design}

The channels are assumed to be slowly varying frequency-selective Rayleigh fading channels, decided by the model used in [2]. We assume that each subcarrier signal experiences flat Rayleigh fading, while the $S$ number of subcarriers used for F-domain spreading and for conveying the same STS information experience independent fading.

Let $1 \leq K^{\prime} \leq S$ be the number of users sharing the same set of $M_{x}$ number of STS orthogonal codes. We also assume that any set of $M_{x}$ STS orthogonal codes is shared by the same $K^{\prime}$ number of users. Then, when the $K^{\prime} \mathcal{K}_{\max }$ signals expressed in the form of (1) are transmitted over frequency-selective fading channels, the received complex low-pass equivalent signal can be expressed as

$$
R(t)=\sum_{k=1}^{K^{\prime} \mathcal{K}_{\max }} \sum_{g=1}^{T_{x}} \sqrt{\frac{2 E_{b}}{U T_{b}} \frac{1}{S M_{x} T_{x}}}\left(\left[\mathbf{C}_{k} \mathbf{B}_{k}\right]^{T} \mathbf{Q}\right)_{g} \mathbf{H w}
$$

$$
+N(t)
$$

where $(\mathbf{X})_{g}$ represents the $g$ th row of the matrix $\mathbf{X}$ and $N(t)$ is the complex valued low-pass-equivalent Additive White Gaussian Noise (AWGN) having a double-sided spectral density of $N_{0}$.

\section{Signal Detection}

The receiver of Fig.2 essentially carries out the inverse operations of those seen in Fig.1. In Fig.2 the received signal is first down-converted using the carrier frequency $f_{c}$, and then demodulated using FFT based multicarrier demodulation. After FFT based multicarrier demodulation we obtain $U S$ number of parallel streams corresponding to the signals transmitted on $U S$ subcarriers, and each stream is space-time despread using the approach of [1], in order to obtain $L_{x}$ separate variables, $\left\{Z_{u, l}, Z_{u, 2}, \ldots, Z_{u, L_{x}}\right\}_{u=1}^{U S}$, corresponding to the $L_{x}$ data bits transmitted on the $u$ th stream, where $u=1,2, \ldots, U S$, respectively. Following space-time de-spreading, a decision variable is formed for each transmitted data bit $\left\{b_{u 1}, b_{u 2}, \ldots, b_{u L_{x}}\right\}_{u=1}^{U}$ by de-spreading the corresponding variables associated with the F-domain spreading sequences. Specifically, for the case of $L_{x}=M_{x}=T_{x}=2,4$, 8, etc, let

$$
\begin{aligned}
& \mathbf{z}_{u 1}=\left[\begin{array}{lll}
Z_{u, 1} Z_{(U+u), 1} & \ldots & \left.Z_{((S-1) U+u), 1}\right]^{T} \\
\mathbf{A}=\operatorname{diag}\left\{\sum_{l=1}^{T_{x}} h_{u l}^{2}, \sum_{l=1}^{T_{x}} h_{(U+u) l}^{2}, \ldots, \sum_{l=1}^{T_{x}} h_{((S-1) U+u) l}^{2}\right.
\end{array}\right\} \\
& \mathbf{C}=\left(\begin{array}{cccc}
c_{1}^{I I}[0] & c_{2}^{I I}[0] & \ldots & c_{K^{\prime}}^{I I}[0] \\
c_{1}^{I I}[1] & c_{2}^{I I}[1] & \ldots & c_{K^{\prime}}^{I I}[1] \\
\vdots & \vdots & \ddots & \vdots \\
c_{1}^{I I}[S-1] & c_{2}^{I I}[S-1] & \ldots & c_{K^{\prime}}^{I I}[S-1]
\end{array}\right) \\
& \mathbf{b}=\left[\begin{array}{lll}
b_{1, u 1} b_{2, u 1} & \ldots & \left.b_{K^{\prime}, u 1}\right]^{T}, \\
\mathbf{n} & =\operatorname{Re}\left[N_{u, 1}^{\prime} N_{(U+u), 1}^{\prime} \ldots\right. & \left.N_{((S-1) U+u), 1}^{\prime}\right]^{T}
\end{array}\right.
\end{aligned}
$$

Then the decision variable in terms of the first data bit can be expressed as

$$
\mathbf{z}_{u 1}=\sqrt{\frac{2 U E_{b} T_{b}}{S}} \mathbf{A C b}+\mathbf{n} .
$$

Based on (13) the multiuser MC DS-CDMA signals can be detected by invoking different detection algorithms [4]. In the context of the single-user correlation based detector, let $\mathbf{z}=$ $\left[\begin{array}{lllll}Z_{u 1} & Z_{u 2} & \ldots & Z_{u K^{\prime}}\end{array}\right]^{T}$ represent the decision variables. Then, these decision variables are obtained by multiplying both sides of (13) with $\mathbf{C}^{T}$ of (10), which can be expressed as

$$
\mathbf{z}=\sqrt{\frac{2 U E_{b} T_{b}}{S}}\left(\sum_{s=1}^{S} \sum_{l=1}^{T_{x}} h_{((s-1) U+u) l}^{2}\right) \mathbf{R} \mathbf{b}+\mathbf{C}^{T} \mathbf{n},
$$

where

$$
\mathbf{R}=\left(\begin{array}{cccc}
1 & \rho_{12} & \ldots & \rho_{1 K^{\prime}} \\
\rho_{21} & 1 & \cdots & \rho_{2 K^{\prime}} \\
\vdots & \vdots & \ddots & \vdots \\
\rho_{K^{\prime} 1} & \rho_{K^{\prime} 2} & \cdots & 1
\end{array}\right)
$$

is the correlation matrix of the $K^{\prime}$ user signals, while $\rho_{i j}$ represents the correlation factor between user $i$ and user $j$, which can be expressed 


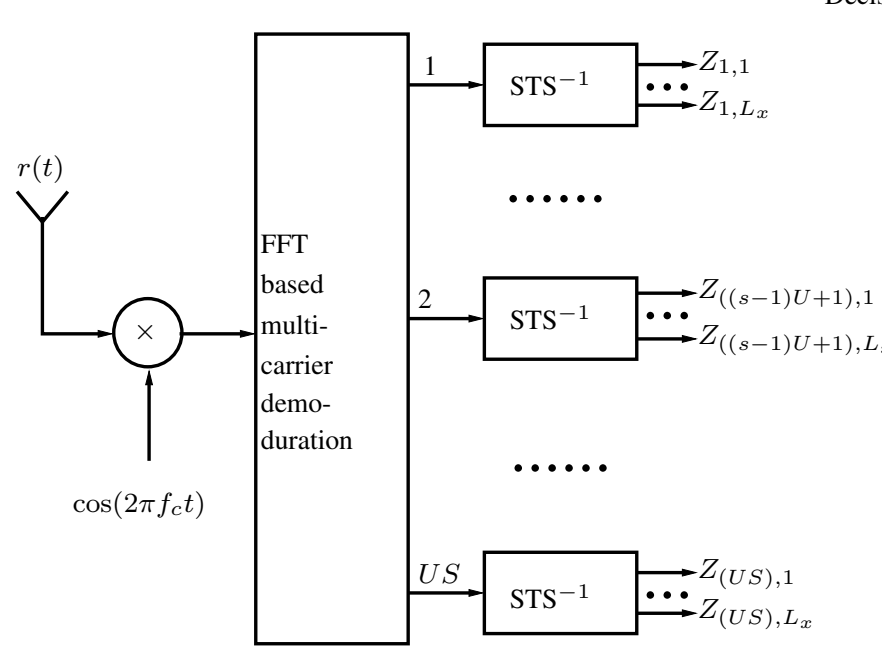

Decision variables for the $u$ th sub-block

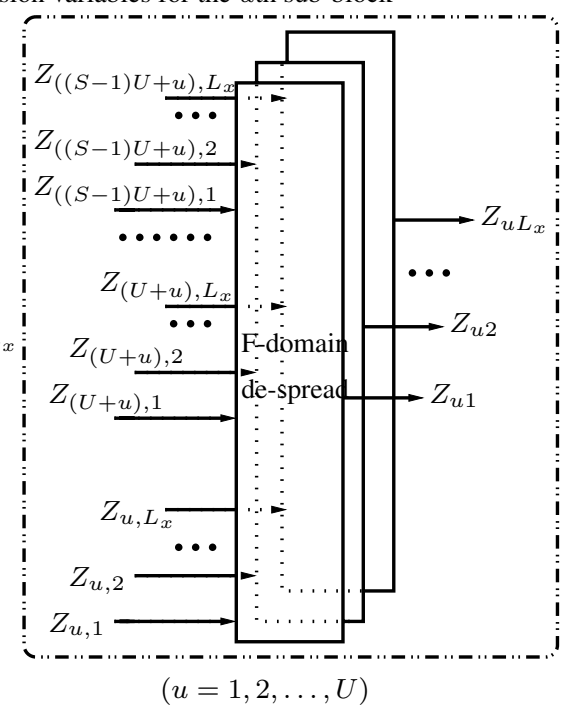

Fig. 2. The receiver schematic of the MC DS-CDMA system using space-time spreading.

as

$$
\rho_{i j}=\frac{\sum_{s=1}^{S}\left(c_{i}^{I I}[s-1] c_{j}^{I I}[s-1] \sum_{l=1}^{T_{x}} h_{((s-1) U+u) l}^{2}\right)}{\sum_{s=1}^{S} \sum_{l=1}^{T_{x}} h_{((s-1) U+u) l}^{2}} .
$$

Equation (14) suggests that the diversity gain contributed both by the transmit diversity and frequency diversity can be retained, since we have a double sum of the components $h^{2}$ corresponding to the transmit and frequency diversity orders of $S$ and $T_{x}$, respectively. However, multiuser interference is introduced, since the channel's time-varying characteristics impair the orthogonality of the sequences $\left\{c_{k}^{I I}[0], c_{k}^{I I}[1], \ldots, c_{k}^{I I}[S-1]\right\}$. Finally, the corresponding data bits, $b_{k, u 1}, k=1,2, \ldots, K^{\prime}$, are decided according to $\hat{b}_{k, u 1}=\operatorname{sgn}\left((\mathbf{z})_{k}\right)$ for $k=1,2, \ldots, K^{\prime}$, where $(\mathbf{z})_{k}$ represents the $k$ th row of $\mathbf{z}$, while $\operatorname{sgn}(\cdot)$ is the sign function [4].

Note that the correlation factors $\left\{\rho_{i j}\right\}$ in (16) are time-variant due to the time-varying nature of the channel's fading envelope. However, since $\sum_{s=1}^{S} c_{i}^{I I}[s-1] c_{j}^{I I}[s-1]=0$, it can be shown that we have $\rho_{i j}=0$, provided that the sum of $\sum_{l=1}^{T_{x}} h_{((s-1) U+u) l}^{2}$ is identical for different values of $s$. Moreover, it can be shown that the correlation factors $\left\{\rho_{i j}\right\}$ are contributed by the differences of the sums $\sum_{l=1}^{T_{x}} h_{((s-1) U+u) l}^{2}$ experienced according to the different values of $s$, while the common part of $\sum_{l=1}^{T_{x}} h_{((s-1) U+u) l}^{2}$ in terms of the different values of $s$ can be successfully removed due to the orthogonality of the F-domain spreading codes. Specifically, let $\sum_{l=1}^{T_{x}} h_{((s-1) U+u) l}^{2}=A_{h}+\Delta_{s}$, where $A_{h}$ represents the average value of $\sum_{l=1}^{T_{x}} h_{((s-1) U+u) l}^{2}$ in terms of $s$, while $\Delta_{s}=$ $\sum_{l=1}^{T_{x}} h_{((s-1) U+u) l}^{2}-A_{h}$. Then, (16) can be written as

$$
\rho_{i j}=\frac{\sum_{s=1}^{S}\left(c_{i}^{I I}[s-1] c_{j}^{I I}[s-1] \Delta_{s}\right)}{\sum_{s=1}^{S}\left(A_{h}+\Delta_{s}\right)} .
$$

In the context of the decorrelating multiuser detector [4], the decision variables associated with $b_{k, u 1}, k=1,2, \ldots, K^{\prime}$ are obtained by multiplying both sides of (14) with the inverse of $\mathbf{R}$, i.e. with $\mathbf{R}^{-1}$, which can be expressed as

$$
\begin{array}{r}
\mathbf{R}^{-1} \mathbf{z}=\sqrt{\frac{2 U E_{b} T_{b}}{S}}\left(\sum_{s=1}^{S} \sum_{l=1}^{T_{x}} h_{((s-1) U+u) l}^{2}\right) \mathbf{b} \\
+\mathbf{R}^{-1} \mathbf{C}^{T} \mathbf{n},
\end{array}
$$

and the corresponding data bits $b_{k, u 1}, k=1,2, \ldots, K^{\prime}$ are decided according to $\hat{b}_{k, u 1}=\operatorname{sgn}\left(\left(\mathbf{R}^{-1} \mathbf{z}\right)_{k}\right), k=1,2, \ldots, K^{\prime}$. Equation (18) shows that each user's data can be decided independently of the other users' data and the diversity order achieved is $T_{x} S$.

\section{Performance Results And CONClusions}

The BER versus SNR per bit, $E_{b} / N_{0}$, performance of both the correlation based single-user detector and that of the decorrelating multiuser detector is shown in Figs. 3 and 4 for the TF-domain spreading assisted broadband MC DS-CDMA systems. In both figures we considered $T_{x}=2$ transmitter antennas and supporting $K=$ $\mathcal{K}_{\text {max }}, 2 \mathcal{K}_{\text {max }}, 3 \mathcal{K}_{\text {max }}$ and $4 \mathcal{K}_{\text {max }}$ number of users corresponding to $K^{\prime}=1,2,3,4$, where $\mathcal{K}_{\max }$ represented the maximum number of users supported by the T-domain orthogonal spreading codes without imposing multiuser interference. As expected, we observe in both figures that the BER performance is significantly improved, when the correlation based single-user detector is replaced by the decorrelating multiuser detector. For both the correlation based single-user detector and the decorrelating multiuser detector, the BER performance degrades, when increasing the number of users sharing the same $\mathrm{T}$ domain spreading code, i.e. when increasing the value of $K^{\prime}$. However, the BER degradation due to increasing the value of $K^{\prime}$ is significantly lower for the decorrelating multiuser detector, than that of the correlation based single-user detector. Furthermore, upon comparing Fig. 3 to Fig.4, we observe that the BER performance of the decorrelating multiuser detector is closer to the BER performance achieved 
without multiuser interference, when using $S=8$ (Fig.4) instead of $S=4$ (Fig.3).

In Fig. 5 we investigated the BER performance of both the correlation based single-user detector and that of the decorrelating multiuser detector for various numbers of transmitter antennas, namely for $T_{x}=1,2,4$ and for $S=8,4,2 \mathrm{~F}$-domain spreading codes, while maintaining a constant $T_{x} S$ value of eight. In our experiments we assumed that $K^{\prime}=2$, i.e. that each set of T-domain STS codes was shared by two users. Let the maximum number of users supported by the T-domain codes be $\mathcal{K}_{\max }$, while using the parameters of $\left(T_{x}=4, S=2\right)$. Then, for a broadband MC DS-CDMA system having a constant system bandwidth the maximum number of users, $\mathcal{K}_{\text {max }}$ supported by the T-domain codes and using the parameters of $\left(T_{x}=1, S=8\right)$ or $\left(T_{x}=2, S=4\right)$ is $\mathcal{K}_{\max } / 4$ or $\mathcal{K}_{\max } / 2$, respectively. In other words, there is a maximum of two, four or eight users sharing the same set of orthogonal STS codes, corresponding to the cases of $\left(T_{x}=4, S=2\right),\left(T_{x}=2, S=4\right)$ or $\left(T_{x}=1, S=8\right)$, respectively. From the results we infer a number of observations. (a) All schemes achieve the same total diversity gain; (b) The number of transmitter antennas has the same effect on the BER performance as the length of the F-domain spreading codes, i.e. the same BER can be maintained, regardless of what values $T_{x}$ and $S$ assume, provided that the product $T_{x} S$ remains a constant; (c) The decorrelating multiuser detector significantly outperforms the correlation based singleuser detector. The gain achieved at the BER of $10^{-3}$ by using multiuser detection instead of single-user detection is about 5-6 dB; (d) The maximum number of users, $K_{\max }^{\prime}$, sharing the same set of STS orthogonal codes is two, four or eight, when we use the parameters $\left(T_{x}=4, S=2\right),\left(T_{x}=2, S=4\right)$ or $\left(T_{x}=1, S=8\right)$, respectively. Furthermore, since according to Figs. 3 , 4 the BER performance degrades upon increasing the number of users sharing the same set of STS orthogonal codes, consequently, for a fully loaded system supporting the maximum number of users, namely $K_{\max }^{\prime}$, we can surmise that MC DS-CDMA employing $\left(T_{x}=4, S=2\right)$ outperforms the scheme using the parameter combinations of both $\left(T_{x}=2, S=4\right)$ and $\left(T_{x}=1, S=8\right)$. Furthermore, the MC DS-CDMA system using the parameters $\left(T_{x}=2, S=4\right)$ outperforms that employing $\left(T_{x}=1, S=8\right)$. In conclusion, the above arguments suggest that the best broadband MC DS-CDMA system will only use transmit diversity and no frequency diversity at all, i.e. use the parameters $\left(T_{x}=8, S=1\right)$, which simultaneously suggests that no multiuser detection is required.

\section{REFERENCES}

[1] B. Hochwald, T. L. Marzetta, and C. B. Papadias, "A transmitter diversity scheme for wideband CDMA systems based on space-time spreading," IEEE Journal on Selected Areas in Communications, vol. 19, pp. 48-60, January 2001.

[2] L.-L. Yang and L. Hanzo, "Space-time spreading assisted broadband MC DS-CDMA," in Proceedings of IEEE VTC'2002, Spring, (Birmingham, Alabama, USA), May 2002.

[3] E. A. Sourour and M. Nakagawa, "Performance of orthogonal multicarrier CDMA in a multipath fading channel," IEEE Transactions on Communications, vol. 44, pp. 356-367, March 1996.

[4] S. Verdu, Multiuser Detection. Cambridge University Press, 1998.

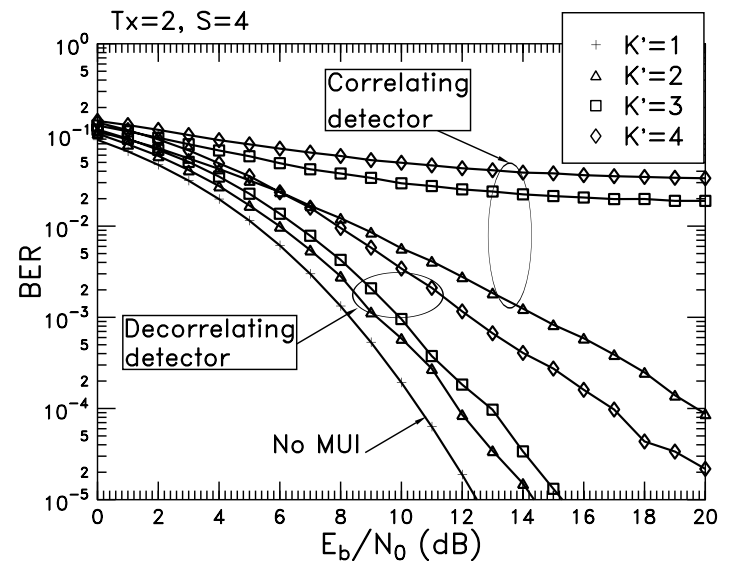

Fig. 3. Simulation based BER versus the SNR per bit, $E_{b} / N_{0}$, performance of both the single-user correlator and decorrelating multiuser detectors for STS based broadband MC DS-CDMA using TF-domain spreading, when communicating over frequency-selective Rayleigh fading channels.

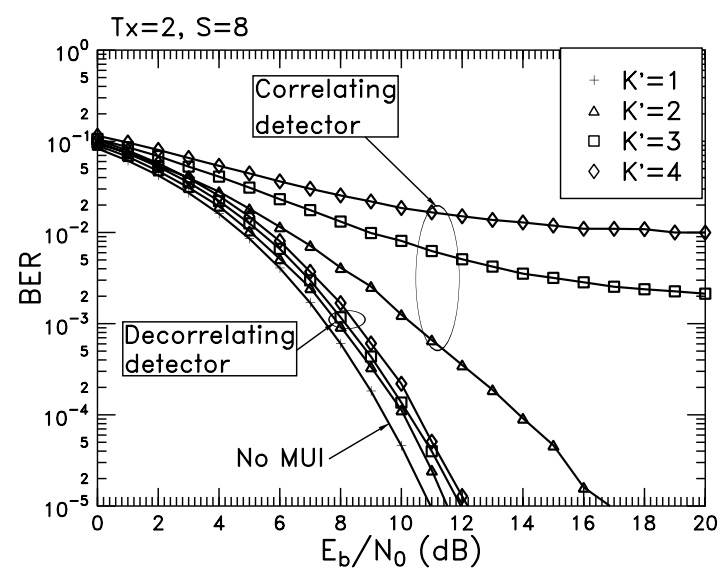

Fig. 4. Simulation based BER versus the SNR per bit, $E_{b} / N_{0}$, performance of both the single-user correlator and decorrelating multiuser detector for STS based broadband MC DS-CDMA using TF-domain spreading, when communicating over frequency-selective Rayleigh fading channels.

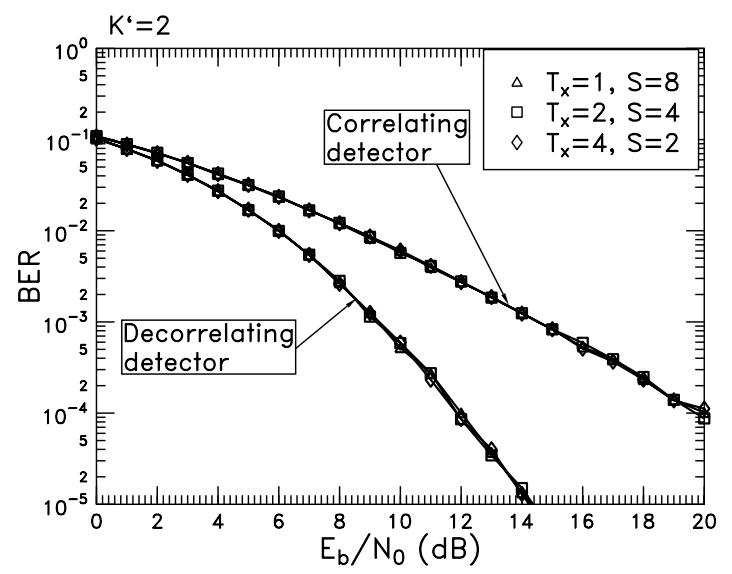

Fig. 5. Simulation based BER versus the SNR per bit, $E_{b} / N_{0}$, performance of both the single-user correlator and decorrelating multiuser detector for STS based broadband MC DS-CDMA using TF-domain spreading, when communicating over frequency-selective Rayleigh fading channels. 